\title{
The Design And Implementation Of USB Mass Storage And CCID Composite Device
}

\author{
Wei Zhang ${ }^{* a}$, Jinnan Zhang ${ }^{\mathrm{a}}$, Yang’an Zhang ${ }^{\mathrm{a}}$, Bin Lu ${ }^{\mathrm{a}}$ \\ ${ }^{a}$ State Key Laboratory of Information Photonics and Optical Communications (BUPT), P.O. Box 66 \\ \#10 Xitucheng Road, Haidian District \\ Beijing 100876, China \\ J_weiweimao@163.com
}

\begin{abstract}
USB disk and UKEY are common applications of USB Mass Storage and CCID protocol and used widely in our daily life, but they are always deployed on different kinds of devices such as USB disk and UKEY. A potential application that integrates the functions of these two protocols into one device is discovered by us. In this way, we can regard a single device as a mass storage and an UKEY as well. According to this discovery, a new Mass Storage and CCID composite device is developed on IS8U192A development board. After this, several tests are made with some test tools to ensure the newly developed device is able to function well and the Mass Storage and CCID protocols are accurately implemented.
\end{abstract}

Keywords-component; USB; CCID; Mass Storage; Composite Device

\section{INTRODUCTION}

As the rapid development of electronic commerce, more and more demand for the security and convenience when we trade through the Internet. The UKEY to the trade that happened in the internet likes the Firewall to our PC, keeping us in a safety environment during the trading. CCID protocol is standard of smart card reader device applied to the UKEY, while USB Mass Storage protocol applied to traditional USB disk device used to store materials. Just for the UKEY being used, we must install corresponding driver on our own PC, to make UEKY work normally. We may wonder, why not mix USB disk and UKEY as one device, and make full use of their functions, like mass storage device store driver, in this way, this mix device can plug and play. Based on this idea, we want to make a composite device. This article aims to develop a new composite device: Mass Storage + CCID.

\section{SOFTWARE AND HARDWARE}

As we all know, USB is the short of Universal Serial Bus Specification, USB Mass Storage is the standard of mass storage device. Mass Storage protocol, not only defines the physical characteristics of the USB device, like data flow model, electrical, also defines some normal behaviors, like suspend, reset, hub configuration and so on [1]. CCID is smart card reader specification that defines the packet types, formats in the communication between ICCs and host, and PIN modification in order to strengthen the security and so on [2].
After learning the protocols, we also need to learn the hardware system structure of development board named IS8U192A, which mainly applied to make UKEY. It's based on the HED HC-8051 8bit CPU, provides two working modes: full-speed and low-speed, five endpoints for communication include one bilateral control endpoints, 4 Bulk/Interrupt endpoints, and supports double buffer function. The control endpoint used in the recognition and configuration process after device plug into host. As for the rest of four Bulk/Interrupt endpoints, two used for input, the others used for output. The address space of IS8U192A can be divided into firmware area, user program area, security algorithm area, CPU RAM, MPU, especially MPU contains CPU RAM, ROM, and flash. In the process of development, we mainly use user program area and flash.

\section{DEVELOPMENT PROCESS}

A. Struture

Before software development, we should design the whole development structure of composite device first.

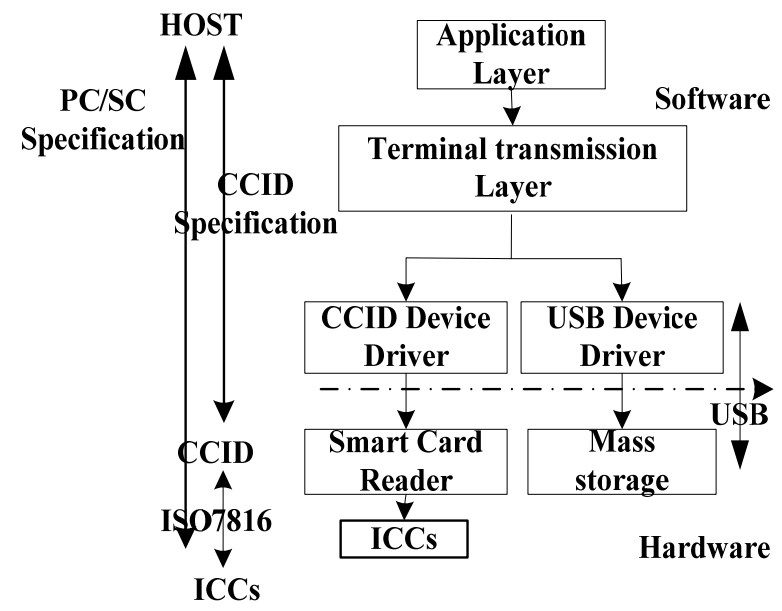

Figure 1. Composite device application structure

Figure 1 is the overall application structure, shows the composite device's position in computer software and hardware, what's more, also shows the position of corresponding protocols. USB Mass Storage and CCID 
protocols have been learned on the above paragraph, ISO7816 is the specification which defines the interaction between ICCs (smart card) and host. In the development of composite device, we just realize the function of CCID protocol and no need to learn specific interaction content between ICCs and host. If the device has been learned by host on device characteristics like device type, distribution communicate address and so on, then when application layer asks some requests, driver program will according the specific request then decided to send to some designated device. In the CCID development, we analysis corresponding data or command according to the communication between host and ICCs, so as to realize the corresponding command operation and related data transfer. While in the Mass Storage development, mainly concern bulk-only transmission. The protocol's realization and code merge are the keys of composite device development process.

\section{B. Software Development}

1) Enumeration: After a new device plug into the host, enumeration is the first action of the host to device, in order to learning the characteristics of the new device [3].

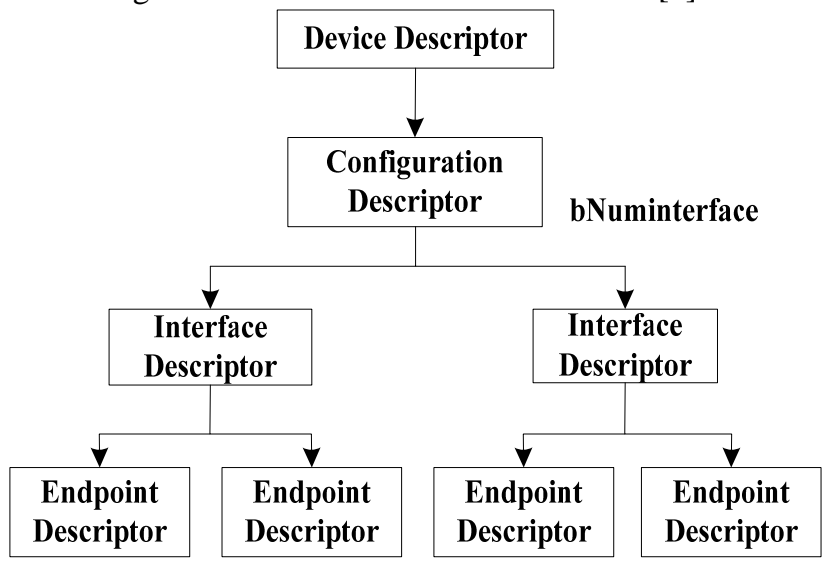

Figure 2. Eumeration's architecture of composite device

For a composite device, the sequence of enumeration is same as ordinary device. Look at Figure 2, it's a simple logical hierarchy chat for the enumeration of composite device, from it, we not only see the enumeration process's order, still can notice, there're two interfaces, one for Mass Storage and another for CCID, so bnuminterface field in the configuration descriptor should be set value 2 , it is the place that different with ordinary device's enumeration. Mass Storage device and CCID device have their own interface descriptor and endpoint descriptor respectively.

2) Reuse of Endpoints: Before protocol development, we need to solve two problems: endpoint distribution and imitate ICCs. IS8U192A development board provides five endpoints for us to dominate: two Bulk/Interrupt endpoints for IN direction, two Bulk/Interrupt endpoints for OUT direction, and one control endpoint, which used in enumeration process, cannot be used with reflex. Because mass storage device, must have two endpoints for Bulk-only transmission, thus CCID device with only two available endpoints. So in order to realize composite device, we need to imitate ICCs, such we can use less endpoint in CCID protocol realization. Just simulate the existence of ICC card, no need to analog the specific command content in the interaction between ICCs and host.

Development of Protocols: According to the USB2.0 protocol and some related specifications, we can divide development into three parts. First realize enumeration process. Second realize commands belong to USB class, include GET MAX LUN and BULK-ONLY mass storage RESET [4]. GET MAX LUN command issued by host sends to device asks for how many logical units does device have. BULK-ONLY mass storage RESET mainly reset device and some related interfaces. After finish those, host will go into the bulk-only way transmit to device [5]. Third step is to realize SCSI commands that is INQUERY, READ CAPACITY, WRITE(10), READ(10). At this stage, all commands, state, and data are exists in the two forms of data packet, respectively is CBW (Command Block Wrapper) and CSW (Command State Wrapper) [6].

Because ICCs has been simulated on the smart card reader device, we just use two Bulk endpoints and Bulk-only transmission to realize the interaction and then realize the function of CCID protocol.

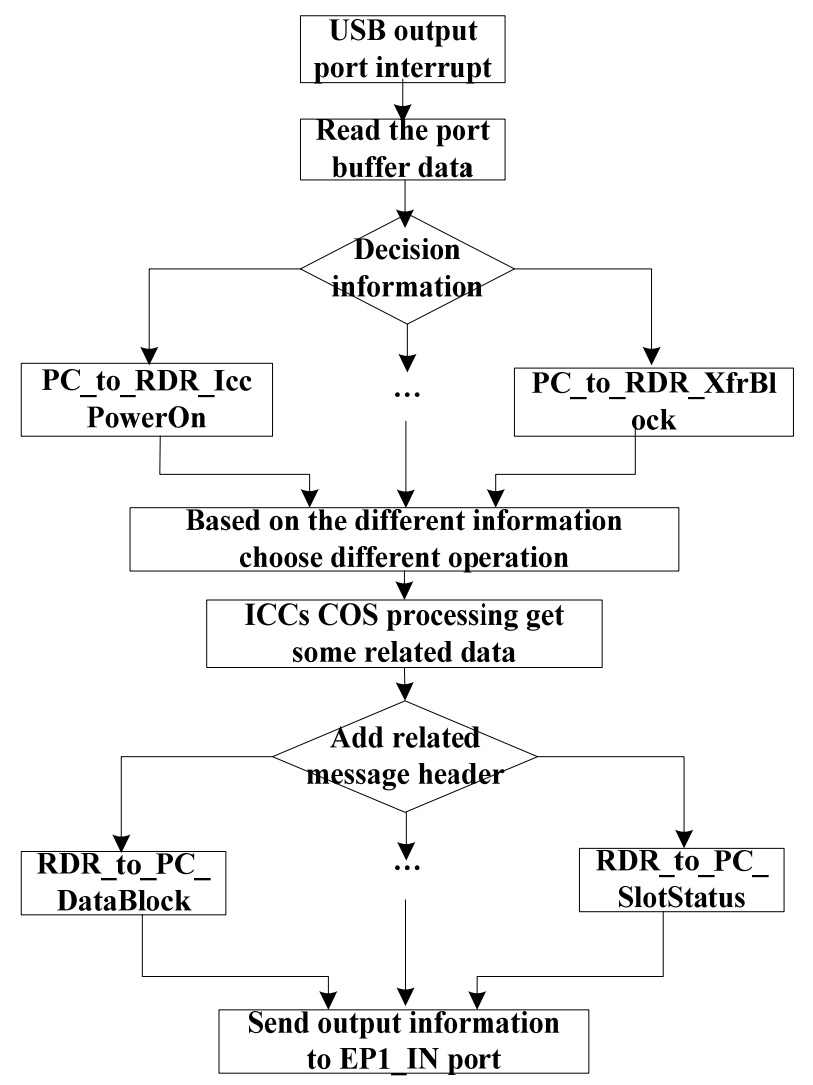

Figure 3. CCID overall process flow chart

Figure 3 shows the overall process of CCID protocol handing message process. Development idea is go into the 
interrupt handling function in a way of USB interrupt [7]. First, interrupt handling function will obtain data from the buffer of endpoint. After recognition the acquisition data, and compared with some related corresponding CCID message, according the result of contrast to choose different operations. Then analyze data or command of the rest in the buffer in the same way. CCID protocol likes a bridge in the interaction, either analysis the command issued by host then transfer to ICCs, or add message header to the processed data by ICCs cos, package them into CCID message format send to host.

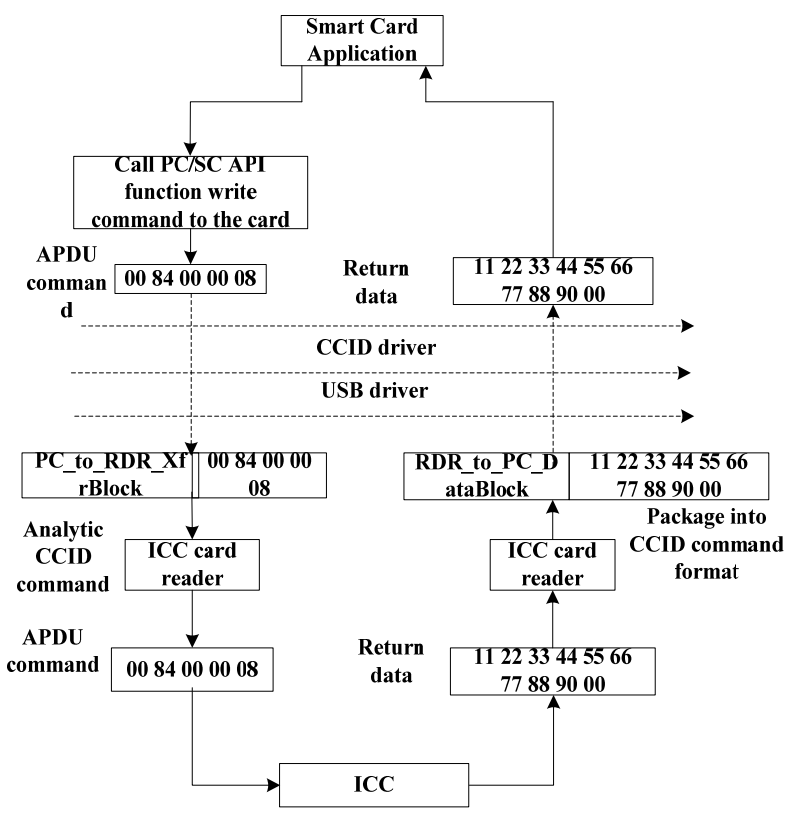

Figure 4. Smart Card application system communication model

Figure 4 is a traffic model of smart card applications, mainly describes the change of related data formats in the interaction, and the role of smart card reader, in the aid of PC/SC API function, host sends APDU command to the smart card.

\section{Test}

After the completion of software development, followed by test include function of protocols and composite device. We mainly test the integrity and robustness of the Mass Storage protocol using a tool named USBlyser, that is used to test USB Mass Storage functional specially and provided by www.usblyser.com [8]. The test content include: enumeration and SCSI commands. Left picture in the Fig. 5 shows the result of this test. Left green section is the specific test items and the green color means test items are standard. For the CCID protocol, there's no special test tool can be used, so we write test software by ourselves mainly test the function of the CCID protocol.

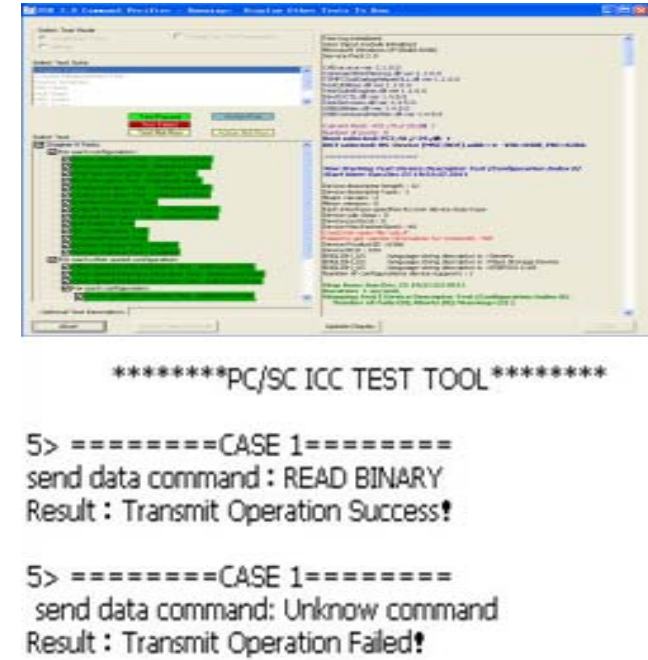

Figure 5. Test result screenshot of USB mass storage and CCID protocol

Right picture in the Figure 5 is the test result of CCID protocol. We choose command named READ BINARY as test case, using software designed by ourselves. In this test, we entered right and wrong APDU command respectively, aim to test the correctness of the CCID protocol.

D. Processors

Smart card readers

Microsoft Usbccid Smartcard Reader (WUDF)

D. If Sound, video and game controllers

A. Storage controllers

- Intel(R) Desktop/Workstation/Server Express Chipset SATA RAID Controller

1) System devices

4. Universal Serial Bus controllers

Generic USB Hub

Generic USB Hub

Generic USB Hub

Intel(R) 6 Series/C200 Series Chipset Family USB Enhanced Host Controller - 1C26 Intel(R) 6 Series/C200 Series Chipset Family USB Enhanced Host Controller - 1C2D

USB Composite Device

USB Mass Storage Device

USB Root Hub

USB Root Hub

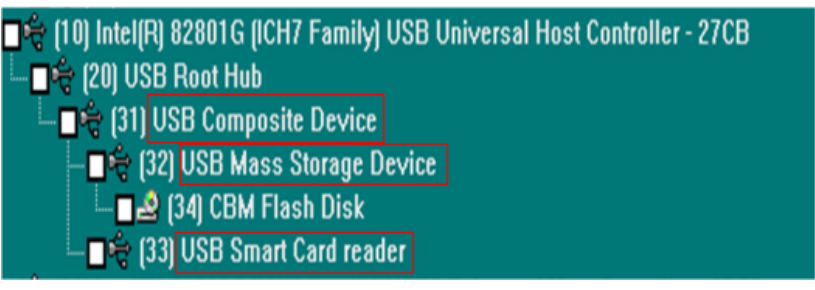

Figure 6. Test result screenshot of composite device

In the Figure 6, the above picture is the screenshot of device manager, the below picture is the screenshot of Bushound when a composite device plug into host first. It can state composite device development is successful, the operating system can identification this device normally.

\section{CONCLUSION}

We realize the composite device (USB Mass Storage and CCID) on the development board named IS8U192A mainly used by UKEY with only five endpoints successfully. 
What's more, this composite device is a single device with two functions of USB disk and UKEY. The successful realization of this application (USB Mass Storage and CCID composite device) makes UKEY get rid of the limitation of $\mathrm{PC}$ and driver when been used, because the driver can be stored in USB disk, makes UKEY change into a plug and play device, makes UKEY more popular and get more extensive application in the future. With the development of composite device and integrated circuits mature, there will be more variety composite devices, improve the living more efficiency and more flexibility constantly.

\section{ACKNOWLEDGMENT}

The work is supported by the Fundamental Research Funds for the Central Universities (2009RC0401), the fund of young scholar innovation project (2001PTB-00-31), the fund of young scholar innovation project (2012RC0407), the fund of young scholar innovation project (2012RC0406).

\section{REFERENCES}

[1] Universal Serial Bus Specification, Revision 2.0, April 27, 2000

[2] Universal Serial Bus Device Class: Smart Card CCID Specification for Integrated Circuit(s) Card Interface Device, Revision 1.1, April 22rd, 2005.

[3] QIAN Hao: The Process and Code Design for USB Enumeration. Computer Knowledge and Technology Vol.6, No.19, July 2010, pp. 5269-5272.

[4] Universal Serial Bus Mass Storage Class Control/Bulk/Interrupt (CBI) Transport, Revision 1.1, June 23, 2003.

[5] Universal Serial Bus Mass Storage Class Specification Overview, Revision 1.2, June 23, 2003

[6] Universal Serial Bus Mass Storage Class Bulk-Only Transport, Revision 1.0, September 31, 1999

[7] Rong LIU, Teach you play USB (Beijing University of Aeronautics and Astronautics Press January 1, 2009).

[8] Information on www.usblyser.com

[9] Jinnan Zhang,Yue Gu,Xueguang Yuan,Feng Tian,Xiaoguang Zhang and Jinjing Tao,"A new control algorithm for automatic PMD compensation system”, J.Chin. Opt. Lett. , Vol.10 , Issue 03 . 